Future lines of enquiry should include an objective analysis of the work of individual members, and satisfaction questionnaires regarding aspects of their work outside of the team meeting.

\section{Acknowledgements}

We are grateful to Dr Dinshaw Master for help with this study.

\section{References}

Cockburn, J. J. (1989) Clinical decisions about patients. Management within multidisciplinary clinical teams. Psychiatric Bulletin, 13, 130-134.
Coles, R. J., Von Abendorf, R. \& Herzberg, J. L. (1991) The impact of a new community mental health team on an inner city psychogeriatric service. International Journal of Geriatric Psychiatry, 6, 31-39.

Donabedian, A. (1988) The quality of care. How can it be assessed? Journal of the American Medical Association, 260, 1743-1748.

OvRetvert, J. (1990) Making the team work! Professional Nurse, 5, 284-288.

Royal College of Psychiatrists (1977) The responsibilities of consultants in psychiatry within the national health service. Bulletin of the Royal College of Psychiatrists, September, 4-7.

SoNI, D. S. (1989) Multidiscplinary teams and line management. Practical problems and areas of conflict in clinical psychiatry. Psychiatric Bulletin, 13, 657-661.

\title{
Psychiatry of learning disabilities
}

\author{
Peter K. Carpenter, Secretary, South Western Region Learning Disabilities \\ Audit Group and Consultant Psychiatrist, Hanham Hall Hospital; and \\ Sunita Kanagaratnam, Senior Registrar, Hanham Hall Hospital, Hanham, \\ Bristol BS15 3PU
}

Medical audit has become an overt part of our work over the last few years, hastened by the 1989 White Paper. At a meeting on 1 November 1989, the mental handicap psychiatrists of Bristol and Weston, Cheltenham, Frenchay, Gloucester, and Southmead Health Authorities agreed to meet monthly to audit agreed topics in mental handicap across district boundaries. These meetings were to be in addition to any local district audit that may already have been occurring, and were to provide a peer group for audit and a means of cross-fertilisation of ideas for projects. The group has now operated for over two years, and would like to present some examples of the topics covered and some comments on its experiences to help stimulate correspondence in the Psychiatric Bulletin on medical audit in learning disabilities.

\section{The study}

\section{Anticonvulsants}

The initial standard set was that all patients with epilepsy should be maintained with clinically acceptable seizure control on one anticonvulsant. The in-patients on more than one anticonvulsant were looked at in the group and the consultants asked to justify the therapy. In addition, an initial blood monitoring standard was agreed of six monthly checks of drug levels, full blood count [FBC], liver function test [LFT] and urea and electrolytes [U\&E], with three monthly U\&Es for patients on acetazolamide.

The follow-up meeting again peer reviewed patients on polytherapy. The blood monitoring standard had so universally failed that no-one felt able to assess its merit! We discovered that we had to improve our routine call-up systems and felt we should still aim at the six monthly standard. The third meeting of the cycle found this still to be a major failing, which was frustrating as many of the blood tests available were abnormal. This cycle looked at fit control as well as polytherapy, and suggested that patients having more than ten seizures in six months should have their therapy reviewed before the next meeting in the cycle.

\section{Down's syndrome}

The initial meeting discussed what baseline and routine ongoing screening each district performs and their apparent value. It was agreed to set an initial minimum standard of an annual check of thyroid function, FBC, U\&E and LFT. There was indecision on how frequently routine physical examinations should be done. 
The follow-up meeting found most districts met these standards. There were sufficient data from the blood tests and routine physical examinations for the group to agree a revised minimum standard of annual checks of FBC, thyroid function, and ears. The group resolved to continue to collect data on adverse physical health events in this group to monitor the standard's usefulness as well as its achievement.

\section{Discharge procedures}

The initial meeting discussed the wide variety of procedures used to discharge or transfer long-stay patients. The group felt that it could not audit except by peer review the reasons for discharge, and that minimum standards could be set on the process of discharge. The first minimum standard set was that every discharged patient should have an individual programme planning meeting, should have a full discharge letter received by the accepting medical officer within one week of discharge, that two weeks medication should be supplied, and that there should be formal follow-up by the home team three months after discharge. The follow-up meeting added that next-of-kin and all wards must be notified of the move, and that the patient should have had a full physical examination within the previous two months. A checklist of these standards was made for insertion in each patients notes to make the task of monitoring these standards easier for the follow-up meeting.

\section{Seclusion}

The initial meeting reviewed all patients secluded in the previous year, and compared the different seclusion policies. The policies were very similar and incidence of seclusion seemed to be low. This patient review re-occurred at the follow-up meeting, and had fewer patients to discuss. It was felt that the topic should be developed by examining how accurately seclusion procedures had been followed. The group decided to draft a model seclusion policy acceptable to all districts that could be used as the basis of audit. This is now in final draft stage, and is being submitted to the Mental Handicap Specialist Advisory Subcommittee for comments prior to local approval.

\section{Comments}

This audit group has now been operational for over two and a half years. The initial decision was that the group's first priority would be to gain confidence with the audit process, namely the cycle of setting standards, measuring performance against those standards, and reviewing the standards. The topic content and actual standards set have been of less importance than getting the process established. We started with little objective data for our patient group on which to set our minimum standards, and would not claim that they are definitive. They will only remain unaltered if our audit process is ineffective.

The group has learnt the hard way that some topics are too big to fit into the cycle in their original formulation and that it is better to start on a small area of a topic and expand. Early on the group looked at "reasons for admission". The group quickly discovered that some districts had highly incomplete data, and that the range of community support services and admission facilities available were so varied that we did not know where to start in setting minimum standards that we could expect to monitor accurately.

Our experience has been that it is useful prior to initial topic meetings for someone to formally review the literature on the subject and to present a suggested means of audit to help lead the discussion and make it more pertinent. Similarly it is very useful to do a small review audit shortly after the main audit meeting on a topic to ensure that standards are being followed, and catastrophic failures avoided.

In some more varied areas such as discharges or transfers to general hospitals, we have tended to concentrate on the processes involved and not in subjective opinions on the reasons for the event. Looking at the reasons will come later.

There was comparatively little peer review of cases in the initial period and this has become more common only as the group has settled in. Undoubtedly some of the statistical work initially done was less threatening. However, do not undervalue the power of having work presented to others. Its power to improve practice is out of all proportion to the criticism the work actually receives from peers.

It has probably taken at least a year for the group to settle into working together. The group has, however, maintained an important role. Attendance has been consistently high, encouraged perhaps by the placing of the meeting after an academic meeting (which has outside speakers) and the formal teaching tutorials for the senior registrars.

We would like to hear of the experiences of other audit groups for learning disabilities, and would welcome correspondence on specific topics. 